\title{
DEMOCRACY AND CITIZENSHIP
}

\section{IN DIGITAL SOCIETY}

\section{SOCIAL MEDIA USE \& POLITICAL ENGAGEMENT IN DENMARK // REPORT 2016}

Luca Rossi // Sander Schwartz // Martina Mahnke

\section{MAIN FINDINGS OF THE SURVEY}

» Social media use is a daily practice in Denmark, however, frequency and type of use differ greatly.

" Danes use social media primarily to read content; it is less frequently used for producing original content or for interacting with content produced by others.

»Younger Danes are more active and present on social media platforms than older generations. The generation between 20 and 39 years is most likely to use Facebook in order to discuss politics with strangers.

» When specifically looking at how users understand their communication on Facebook, it turns out that many of them view their communication as private. Especially the social network Facebook is used for private communication, e.g. exchanging messages with close friends and family.

» In general, it is not very common for Danish citizens to actively engage in political debates online with strangers, and to change a personal view on a political issue after this. Nevertheless, there is a smaller group of Danes who say they do so.

The Danish population has always been quick to adopt new technologies. When Facebook was first introduced to a broader public, Denmark quickly became a country with one of the highest numbers of adopters in the world. The fact that Danish citizens are so present on social media makes Denmark a unique place when it comes to studying the potential of social media platforms as democratic spaces for public debate.

An important finding is that the Danish interest in social media does not transfer to all social media platforms equally. Twitter has not reached anywhere near the same level of popularity as Facebook, however, it has gained certain popularity in some communities particularly within politics and media. Further, the presented study shows that Danes use social media platforms mostly for reading rather than producing original content. This serves as an important reminder that everyday use of social media platforms is more often related to reading activities than original content creation. Ongoing discussions have highlighted the democratizing potential of social media or in contrast critiqued social media for contributing to the deterioration of the public sphere. The findings of this survey do not confirm either of these two extreme positions. Instead, this study shows similarities to what is already known about offline social interaction ${ }^{1}$, namely that people in general are not very open towards discussing politics in public settings. The fact that our study confirms this for social media should not be interpreted as a failure of the democratic potential of social media; instead we need to understand social media use as an extension of offline interactions. Social media is groundbreaking in its potential of enabling people to interact in a semi-public setting across time and space. However, as social media platforms become more familiar and part of our everyday practice, it is only natural that its usage also reflects generally known social behavior.

\begin{tabular}{llll}
\hline Social Media & Account & Daily users & Daily users (all) \\
\hline Facebook & $72.4 \%$ & $74.2 \%$ & $58 \%$ \\
Twitter & $13.1 \%$ & $6.1 \%$ & $4,9 \%$ \\
Instagram & $15.8 \%$ & $10.5 \%$ & $8,2 \%$ \\
Snapchat & $14.9 \%$ & $12.6 \%$ & $9,85 \%$ \\
Linkedln & $25.9 \%$ & $3.6 \%$ & $2,82 \%$ \\
\hline
\end{tabular}

Table 1: Social media accounts and frequency of use in Denmark (Based on the questions: "On what social media do you have an account?" and "How often do you use the following social media?"; $n=1593$ ) 


\section{Social media: An integral part of everyday Danish life}

Social media such as Facebook, Twitter and Instagram have become an integral part of everyday life in Denmark. Nevertheless, as one would expect, differences exist both internally, between various groups of users within Denmark, and externally compared to other countries. On a national level Denmark is a Facebook loving country while Twitter is mostly relevant to a particular group of media and political experts. This difference between the generally very well known social network site Facebook and the microblogging service Twitter is interesting, if compared with i.e. US data. According to the presented survey $13 \%$ of the Danish population have activated a Twitter account; in the US a PEW ${ }^{2}$ survey reports $23 \%$ of registered Twitter accounts. In comparison, when looking at Facebook the difference is reduced to zero. In both countries $72 \%$ of the population have an account.

Observing the demographics of Danish social media users, it is possible to obtain a more detailed picture of what is currently going on. While social networks like Facebook show a broad user base, at least in terms of age, other services, such as Instagram or Snapchat are used more by specific parts of the population. Snapchat is the most visible case with a remarkable $82 \%$ of users in the age group 16 to 19 and $52 \%$ in the next group (20 to 29). In comparison, Facebook is used by $91 \%$ of users in the first group and by $90 \%$ in the latter. This data suggests a dualistic process: on the one side we are witnessing a normalisation of Facebook. Facebook is no longer the "cool new thing" widely adopted only within the younger part of the population; it has arrived in all age groups.

However, this does not mean that Facebook is dead as sometimes suggested or that it will die anytime soon; it shows that Facebook has become an essential part of our everyday life and that this development is no longer limited to the traditional group of early and young adopters. On the opposite side, the presence of social media with clearly identifiable generational traits point to the fact that social media can play a crucial role for specific parts of the population by providing semi-public conversational spaces. Particularly on Facebook there is a wide age distribution with many active, elderly users. However, even on Facebook and to a much greater extend on platforms such as Instagram and Snapchat there is still a strong overweight of young users in the age groups below 30.
The previous paragraphs raise the question of how the various social media are actually used. User practice is a challenging problem and extremely hard to investigate with a quantitative approach. Nevertheless, the survey attempted to measure how much Danish users participate in various types of activities within the various social media we have analyzed. The goal of this measurement is not to classify users based on their activity but to describe how much every single social media platform is used to a) produce original content, b) interact with content produced by others, or c) read/observe content produced by other users but without interacting. These three dimensions have been identified through specific questions for each social media and then summarized into an index that expresses how frequently a specific type of activity is performed. It should be noted that the three types of identified activities do not cover the full spectrum of what users can do on social media and that the three types of activities are not mutually exclusive. That is to say, it is theoretically possible to imagine users that produce original content, interact with content produced by others and read without interacting.

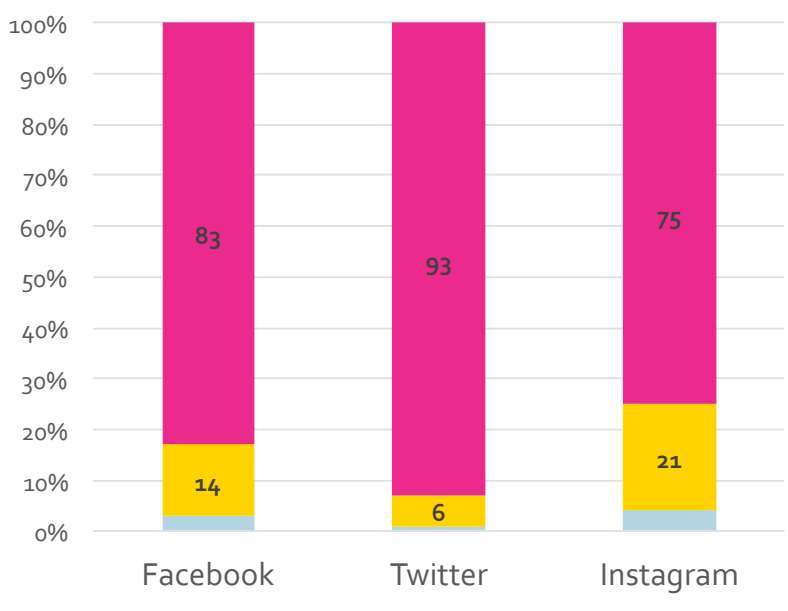

Figure 1: Frequency of original content production on Facebook, Twitter and Instagram ( $\square$ rarely/never, $\square$ less often, $\square$ daily/weekly) (Based on three separate questions: "How often do you use Facebook/ Twitter/Instagram for the following activities ... ?" $n($ Facebook $=1154$, $n($ Twitter $)=208, n($ Instagram $)=252)$

Figure 2 shows the frequency of original content production for Facebook, Twitter and Instagram. Instagram among the three services - is the one that shows a higher frequency of content production (in total $25 \%$ produce content daily/weekly and less often). In comparison, 17\% of users declare to produce original content with a comparable frequency on Facebook and $7 \%$ on Twitter. 
Figure 3 shows a similar picture with regards to the use of social media to interact with content produced by someone else. Further, Instagram shows the highest value with $28 \%$ of the users interacting with other users' content on a daily or weekly base.

Figure 3 shows a similar picture with regards to the use of social media to interact with content produced by someone else. Further, Instagram shows the highest value with

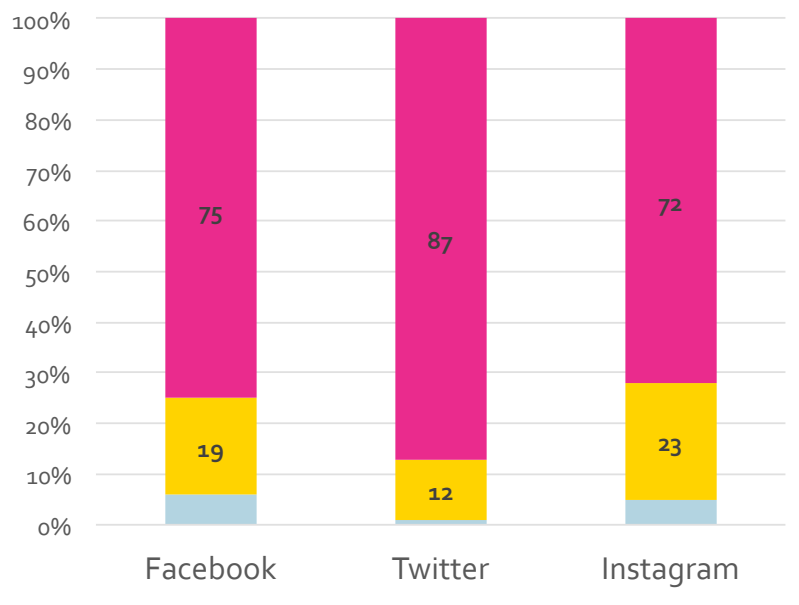

Figure 2: Frequency of how often Facebook, Twitter and Instagram are used to interact with other people's content ( $\square$ rarely/never, $\square$ less often, daily/weekly; based on three separate questions: "How often do you use Facebook/Twitter/Instagram for the following activities ... ?" $n($ Facebook $)=1154, n($ Twitter $)=208, n($ Instagram $)=252)$

$28 \%$ of the users interacting with other users' content on a daily or weekly base.

The combined analysis of Figures 2 and 3 shows a relatively low presence of both original content production and interaction with content. While there are clearly users who do these activities frequently, they do not represent a major part of the whole user base. Within these premises the analysis of Figure 4 is extremely informative. A reading-oriented use of social media is clearly visible through our data with $69 \%$ of Facebook users, $63 \%$ of Instagram users, and $28 \%$ of Twitter users that perform it on a daily or weekly base.

The analysis of the three figures suggest that while more active uses of social media (original content production and interaction) are present in a smaller part of the population, social media is also largely used to read or observe content in a more passive way. This finding is a relevant consequence for social media studies that should be stressed. It shows that social media users in general are a larger and more complex group than the users who make themselves visible through the production of original content or even through the interaction with already available content. Despite the fact that a large part of the academic research on social media has focused primarily on (more or less) active users, it is important to acknowledge the presence of silent readers that do not make themselves visible through content production or high number of interactions.

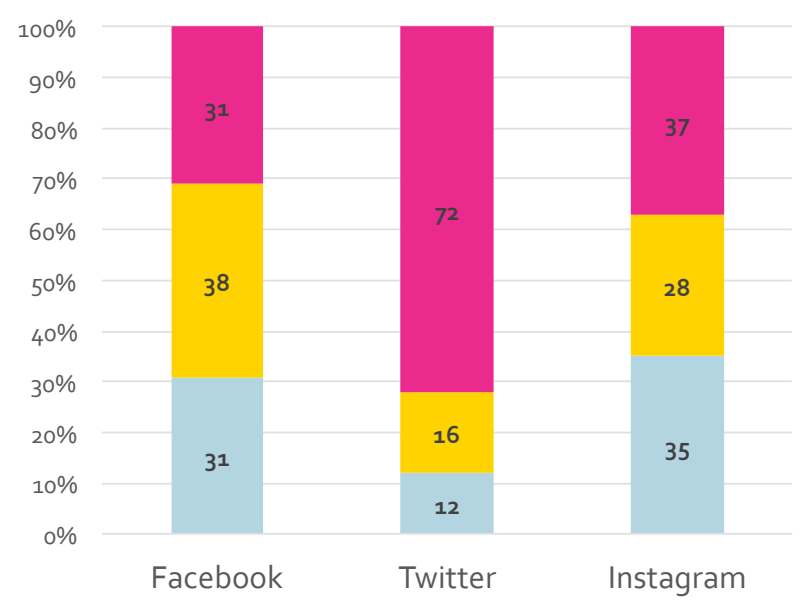

Figure 3: Frequency of how often Facebook, Twitter and Instagram are used to solely read content ( $\square$ rarely/never, $\square$ less often, daily/weekly; based on three separate questions: "How often do you use Facebook/Twitter/Instagram for the following activities ... ?" $n($ Facebook $)=1154, n($ Twitter $)=208, n($ Instagram $)=252)$

\section{Political debate online: Between private mes- sages and public statements}

The following section focusses on another branch of the survey concerned with public political debate on social media. Public debate is defined here as people who discuss politics online with strangers and not private contacts such as friends, family and colleagues on social media. With the popularity of social media many academics have speculated that the internet might produce a more participatory and deliberative public environment for political debate between citizens. Some of these earlier speculations were perhaps too optimistic since we know that people have always been careful about discussing politics and controversial issues in larger social contexts.

Platforms such as Twitter and Instagram are publicly accessible by architecture. Other platforms such as Facebook can be somewhere in between semi-public and semi-private depending on the individual user's privacy settings. Finally, Snapchat is the most private context since every message is usually directed towards a specific receiver or group and disappears after being viewed once. Though early adopters may have used a platform such as Facebook more experimentally in the beginning, it is only logical to assume that the behavior will eventually adapt 
to everyday social behavior. Many platforms such as Facebook and Twitter are maturing and appropriated into the everyday life of Danish citizens. On the one hand, this makes online behavior less experimental and surprising overall; on the other hand it is still important to consider the implications of these new digital conversations and interactions.

One of our main interests in the survey was whether Danes conceive of their online communication as public or private. Personal understanding of privacy on Facebook turns out to be a difficult question, which shows when we asked Danes, using a semantic differential, to vision themselves between two opposite claims: "What I write on Facebook is public communication" and "What I write on Facebook is private communication" (see figure 4). Here, two major groups of respondents can be identified - one of them positioned midway between the two

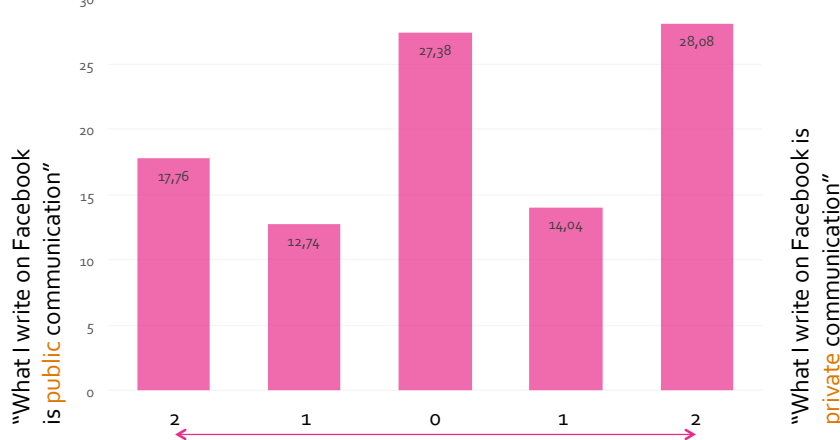

Figure 4: "What I write on Facebook is public/private communication" $(n($ Facebook $)=1154)$

extremes (27\%) and the other positioning clearly toward communication as private ( $28 \%$ ). This shows that a majority of people seem to believe that Facebook communication is leaning towards private. However, the other large group of respondents might also be a group of people ences from good friends and family to lesser known or even strangers online. From other studies we know that people are most comfortable discussing politics with friends in small social settings offline ${ }^{3}$. Political opinions are usually considered to be personal and sensitive information. Many people may also want to avoid sharing political opinions in a larger social setting because it can lead to confrontational behavior and divide social networks. On the other hand, political debate between political groups can be good because it allows people to consider new perspectives of an issue. However, many people may personally prefer to avoid sharing political opinion in public for a variety of reasons including embarrassment and judgement by strangers as well as peers. Relatively few people say that they discuss politics with strangers on social media. Facebook is clearly the most popular place to do so especially considering the people who say they do so often. Out of all respondents, $10 \%$ say they do so often and $31 \%$ say they only do this seldom (see table 2 ). Therefore, Facebook is the social media platform where users are most likely to discuss politics, though it is also important to note that $59 \%$ of Facebook users say they never do so. In comparison, 93,4\% say they do not discuss politics with strangers on Twitter and only $6,6 \%$ say they do so seldom. Instead of Twitter, the second most popular platforms to discuss politics are online newspapers, web fora and email. In online newspapers 3,6\% say they discuss politics with strangers often and $26,3 \%$ do this seldom. On web fora the numbers are $3,4 \%$ and $21,9 \%$. Interestingly we also found a surprisingly large group of people who said they would discuss politics with people they did not know over email. $3 \%$ said they did this often through email and $23,7 \%$ said they did so seldom. A notable finding is that Twitter is one of the least likely places that users who find it hard to define their interactions on Facebook according to a binary understanding of public or private. Looking into age distribution there is a small increase in people who believe their communication on Facebook is not public according to older age. A careful interpretation of this could be that older people are more likely to understand their interaction on Facebook as private, while younger people may have a more complex view of their communication online as neither entirely private or public. Most social media platforms allow people to interact with a range of different audi-

\begin{tabular}{lccc}
\hline \multicolumn{1}{c}{ Platform } & Often & Seldom & Never \\
\hline Facebook & $10,0 \%$ & $31,0 \%$ & $59,0 \%$ \\
\hline Twitter & $0,0 \%$ & $6,6 \%$ & $93,4 \%$ \\
Your Blog & $0,0 \%$ & $4,7 \%$ & $95,3 \%$ \\
Blogs & $1,7 \%$ & $15,7 \%$ & $82,6 \%$ \\
Email & $3 \%$ & $23,7 \%$ & $73,4 \%$ \\
Online newspaper & $3,6 \%$ & $26,3 \%$ & $70,1 \%$ \\
Forum & $3,4 \%$ & $21,9 \%$ & $75,3 \%$ \\
Other & $2,0 \%$ & $15,0 \%$ & $83,0 \%$ \\
\hline
\end{tabular}

Table 2: How often users discuss politics online with strangers (Based on the questions: "How often do you discuss politics with others, who are not your friends, family or colleagues through one or more of the following channels?"; $n=1593$ ) 


\begin{tabular}{cccccc}
\hline Age & Daily & Frequently & Occasionally & Rarely & Never \\
\hline 16 to 19 & $0,0 \%$ & $0,0 \%$ & $14,3 \%$ & $28,6 \%$ & $57,1 \%$ \\
\hline 20 to 29 & $0,0 \%$ & $22,6 \%$ & $6,4 \%$ & $32,2 \%$ & $38,7 \%$ \\
\hline 30 to 39 & $0,0 \%$ & $16,2 \%$ & $16,2 \%$ & $32,4 \%$ & $35,1 \%$ \\
\hline 40 to 49 & $1,7 \%$ & $12,3 \%$ & $10,5 \%$ & $19,3 \%$ & $56,1 \%$ \\
\hline 50 to 59 & $1,8 \%$ & $8,3 \%$ & $7,4 \%$ & $25,9 \%$ & $56,5 \%$ \\
\hline 60 to 69 & $0,0 \%$ & $6,7 \%$ & $5,9 \%$ & $23,0 \%$ & $64,4 \%$ \\
\hline$>70$ & $1,0 \%$ & $6,2 \%$ & $5,2 \%$ & $19,8 \%$ & $67,7 \%$ \\
\hline
\end{tabular}

Table 3: How often users discuss politics on Facebook with strangers (according to age) (Based on the questions: "How often do you discuss politics with others, who are not your friends, family or colleagues through one or more of the following channels?"; $n=1593$ )

Further, we asked how many people have changed their view after a discussion with strangers online. $55 \%$ say they have never changed their view after a discussion, which means $45 \%$ acknowledge that they have done so at one point from these discussions. Only $5 \%$ say they do so often. The largest group of people who say they have changed their opinion after a discussion online is the $25 \%$ who say this happens rarely. In conclusion,

will start a political debate with strangers, which is based partly on the fact that the platform is not popular within the Danish population as a whole.

According to this study, Facebook is the most likely place to discuss politics with strangers overall. This is somewhat to be expected from the popularity of the platform alone. Online newspapers and blogs usually require users to create unique accounts. The hassle alone of creating a user account on a newspaper site or blog just to post a comment, may discourage many people from engaging in a debate in that context.

In contrast, a debate on Facebook may occur spontaneously as people stumble upon a debate in their newsfeed, while they are already logged in. Hence, it is valuable to look further into the political debate specifically on Facebook. If we consider how often people discuss politics on Facebook with strangers according to age, the most active users are 20 to 39 . These age groups have the highest percentage of people who say that they discuss politics frequently with strangers, and they are also the least likely to say that they never discuss politics with strangers. we see that most people never or rarely change their view online after discussing with strangers. On the other hand we can also see that about $20 \%$ say they change their view after a discussion with strangers online either rarely or more often.

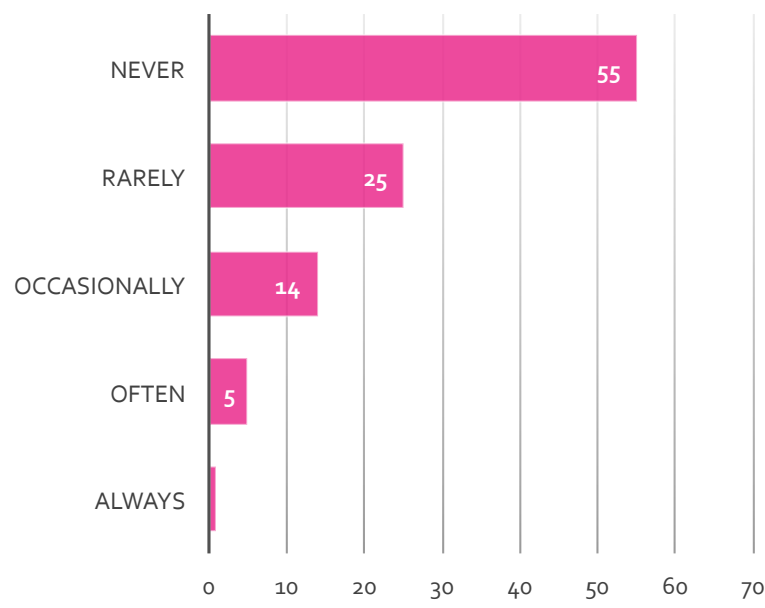

Figure 5: "Have you changed your view on a political subject after having participated in a political discussion online?"

(Based on respondents who did not that they never participated in political discussions with strangers online; $n=279$ )

DECIDIS is a research network based at the IT University Copenhagen. The core objective of the international network is to analyze and understand the ongoing changes in digital society. Given the intense pace of the development of communication technologies and their wide-ranging - if at times equivocal - impact on the way we live, think and experience the world, we believe that research has a major responsibility to keep up with and illuminate the social and cultural transformations this ensues, not at least in terms of their significance for democracy. Thus, the core research objective that unites the work and interests of the participating scholars is to explore ongoing processes in the ways citizens as individuals and in groups interact and act in and with digital society and culture.

More information about DECIDIS: blogit.itu.dk/decidis 